Preprint. Published in:

F. Lamnabhi-Lagarrigue et al. editors,

Taming Heterogeneity and Complexity of Embedded Control

(ISBN: 978-1-905209-65-1), Wiley-ISTE, 2007,

pp. 63-72.

DOI: http://dx.doi.org/10.1002/9780470612217.ch5

\title{
Jurdjevic-Quinn Conditions and Discontinuous Bounded Damping Control
}

\author{
Alex Bombrun - Jean-Baptiste Pomet \\ INRIA Sophia Antipolis, B.P. 93, 06902 Sophia Antipolis cedex, France. \\ Alex.Bombrun@sophia. inria.fr, Jean-Baptiste.Pomet@sophia.inria.fr. \\ ABSTRACT. This note presents a practical stabilization result for discontinuous damping control \\ (or Jurdjevic-Quinn control), in the case where the bound on controls is small. The motivation \\ is to estimate, as that bound goes to zero, how the time taken to reach a neighborhood of the \\ target tends to infinity. \\ RÉSUMÉ. Sous les hypothèses requises, on étudie une version discontinue des lois de contrôle \\ dites "amortissantes" ou encore "de Jurdjevic-Quinn". On présente un résultat de stabilisation \\ pratique lorsque la borne sur les contrôles est suffisamment petite. La motivation est d'estimer \\ sa croissance du temps qu'il faut pour atteindre un voisinage donné de la cible quand la susdites \\ borne tend vers zéro. \\ KEYWORDS: damping control, small control, low-thrust transfer \\ MOTS-CLÉS : damping control, petits contrôles, transfert en poussée faible
}




\section{Introduction}

For smooth control systems whose drift possesses a first integral $V$ which is minimum at some desired configuration (this already makes that configuration Lyapunovstable for the system with zero control), a well known strategy to obtain asymptotic stability, called damping control, or Jurdjevic-Quinn control, consists in using the control to make $V$ decrease; this strengthens stability of the desired configuration, and under some non-degeneracy assumptions, yields convergence, i.e. asymptotic stability. This is recalled in Section 2.

In general, there is a subset $W$ of the state space where $\dot{V}=0$ for any choice of the control, and at each point outside $W$, there is a choice of the control that renders $\dot{V}$ negative (more precisely, at any such point, the control space is separated by a hyperplane; $\dot{V}$ is positive if the control is chosen on one side of this hyperplane, negative if it is chosen on the other side, and zero if it lies in the hyperplane). It is easy to build a smooth feedback that makes $\dot{V}$ negative outside $W$. However, to be continuous, this control must vanish on $W$; in other words, if one insists to design a feedback control that does not tend to zero on $W$, it must be discontinuous on $W$. The closed-loop systems is then an ODE with a discontinuous right-hand side; if one chooses Filippov solutions as the notion of solution of the closed-loop systems (and it makes sense because of measurement errors), it is known that this discontinuous control does not provide asymptotic stability, because at some points of $W$, solutions are not unique, and there appears some spurious equilibrium points, i.e. points where it is possible to "get stuck" instead of converging to the target... these solutions are not mathematical curiosities, but they can be observed, even on simulations, where they appear as "chattering solutions", i.e. very fast oscillations around the position that is idealized as an equilibrium in the sense of Filippov. This can occur only on the set $W$, but it is usually not confined to a neighborhood of the target.

Here, we study precisely the behavior of these discontinuous closed-loop systems, in the case where there is a "small" bound on the control. We are not aware of results in that direction... We prove that, under the usual Jurdjevic-Quinn conditions, we do get "practical" asymptotic stability, and get closer and closer to asymptotic stability as the bound goes to zero. More precisely, for any compact set of initial conditions and any small neighborhood of the target, if the bound on the control is small enough, all (Filippov) closed-loop solutions with these initial conditions go into the neighborhood of the target in finite time and stay in it.

The paper is organized as follow. Section 2 presents the classical Jurdjevic-Quinn method. In Section 3 we introduce a discontinuous feedback, natural in regard of the Judjevic-Quinn conditions and the constraint of small controls and state Theorem 2, our main result. Section 4.1 recalls the invariant principle for differential inclusions due to to A. Bacciotti and F. Ceragioli and Section 4.2 is devoted to the proof of Theorem 2. Motivations are explained in the conclusion. 


\section{Smooth Jurdjevic-Quinn control}

We start with a brief survey of the so called Jurdjevic-Quinn method, which gives sufficient conditions to strengthen the stability property of an affine system which admits a first integral, hence stable in the sense of Lyapunov. We consider the affine system (1) on a smooth manifold $M$ :

$$
\dot{q}=f_{0}(q)+\sum_{i=1}^{m} u_{i} f_{i}(q),
$$

define the distribution $\mathcal{F}^{j}, j \in \mathbb{N}$ by

$$
\mathcal{F}^{j}(q)=\operatorname{Span}_{\mathbb{R}}\left\{f_{0}(q),\left(\operatorname{ad} f_{0}\right)^{k} f_{i}(q), i \in[1,2, \ldots, m], k \in[0 . . j]\right\},
$$

and assume that there exists some integer $l$,

$$
\operatorname{dim} \mathcal{F}^{l}(q)=n \text {, for all } q \in M .
$$

The second assumption is that the drift vector field $f_{0}$, or rather the ordinary differential equation $\dot{q}=f_{0}(q)$ has a periodic solution $t \mapsto \bar{q}(t)$, we denote by $\Gamma$ the compact set

$$
\Gamma=\{\bar{q}(t), t \in \mathbb{R}\} .
$$

It is reduced to a point if the periodic solution is an equilibrium.

The third assumption is that $f_{0}$ has a first integral $V: M \rightarrow \mathbb{R}_{+}:$

$$
L_{f_{0}} V(q)=0 \text { for all } q \in M .
$$

with the following two properties: $V$ is proper, i.e.

$$
\forall \beta \in \mathbb{R}, V_{\beta}=\{q \in M, V(q) \leq \beta\} \text { is compact, }
$$

and it has no critical point outside $\Gamma$ :

$$
d V(q)=0 \Rightarrow q \in \Gamma
$$

Note that these two conditions imply that $V$ reaches its minimum on $\Gamma$. $V$ is constant on $\Gamma$ because it is a first integral of $f_{0}$.

The following theorem is classical, see [SON 98, JUR 78].

Theorem 1 If the conditions (2), (4), (5) and (6) are satisfied, then the smooth feedback $u_{i}^{c}(q)=-L_{f_{i}} V(q)$ asymptotically stabilizes the orbit $\Gamma$.

Its proof relies on applying LaSalle invariance principle [SON 98] to the closed-loop system and the Lyapunov function $V$. It turns out that its time-derivative along solutions is negative except on the set

$$
W=\left\{q \in M, L_{f_{1}} V(q)=0, \ldots, L_{f_{m}} V(q)=0\right\},
$$


and conditions (2), (4), (5) and (6) imply that $\Gamma$ is the only solution of the closed-loop system that remains in $W$.

The Jurdjevic-Quinn idea is to study how the stability properties of the drift, contained in the property $L_{f_{0}} V=0$, may be enhanced by the use of a feedback. Since the original paper [JUR 78] the Jurdjevic Quinn idea has been widely studied and generalized. In particular in [BAC 99] we may find its generalization when the Lyapunov function is not continuously differentiable.

\section{Discontinuous control, main result}

Assume now that there is a "small" bound on the control:

$$
\|u\|=\sqrt{\sum_{i=1}^{m} u_{i}^{2}} \leq \varepsilon .
$$

By small we mean that we are interested in properties that are true for $\varepsilon$ small enough.

The time derivative of $V$ is given by $\dot{V}=\sum_{i=1}^{m} u_{i} L_{f_{i}} V$; the choice $u_{i}=-L_{f_{i}} V$ in the Theorem 1 is one of the many possibilities to make $\dot{V}$ non-positive, and zero only at the points where all the $L_{f_{i}} V$ 's vanish. Especially when the control is constrained, it is natural to optimize this choice in some way.

A "natural" idea is to chose the feedback $u^{\varepsilon}$ which maximizes the absolute value of the time derivative $\dot{V}$ under the constraint (8).

With the notation $\left|L_{h} V\right|=\sqrt{\sum_{i=1}^{m} L_{f_{i}} V^{2}}$, it is well defined, outside the set $W$ defined in (7), by

$$
u_{i}^{\varepsilon}(q)=-\varepsilon \frac{L_{f_{i}} V}{\left|L_{h} V\right|}(q), 1 \leq i \leq m, q \notin W
$$

but it is clearly discontinuous on $W$. At this stage it is useless to define $u^{\varepsilon}(q)$ for $q$ in $W$. The closed loop system then reads $\dot{q}=h^{\varepsilon}(q)$ where the discontinuous vector field $h^{\varepsilon}$ is given by

$$
h^{\varepsilon}(q)=f_{0}(q)+\sum_{i=1}^{m} u_{i}^{\varepsilon}(q) f_{i}(q) .
$$

A Filippov solution of an ordinary differential equation $\dot{x}=f(x)$ with a discontinuous right-hand side, is defined as a solution of the differential inclusion.

$$
\dot{x} \in K f(x)
$$

with

$$
K f(x)=\bigcap_{\rho>0} \bigcap_{\mu(N)=0} \overline{\operatorname{co}}\{f(B(x, \rho) \backslash N)\}
$$


where $B(x, \rho)$ is the ball of center $x$ and radius $\rho$, $\overline{\text { co }}$ denotes the convex closure and $\mu$ is the Lebesgue measure in $\mathcal{R}^{n}$. If the function $f$ is in $L_{l o c}^{\infty}$, it is proved that the multi-valued function $K f$ is upper-semi-continuous locally bounded with nonempty, compact, convex values. Hence for each $x_{0}$ there exists at least one solution of the Cauchy problem $\dot{x} \in K f(x), x(0)=x_{0}$ [FIL 88].

There are many arguments against or in favor of this choice of control. It is clearly the one that causes $V$ to decrease as much as possible at all points. It however has the drawback that, around points where all the functions $L_{f_{i}} V$ vanish, it relies on the direction of an almost zero vector... this makes little sense, and it has been noticed in many places that, in fact, this discontinuous control law does not allow asymptotic stabilization of $\Gamma$ (or of an equilibrium point) because some chattering appears near the target, causing some trajectories to get stuck on some locus instead of converting to that target.

As an example, consider the controlled harmonic oscillator.

$$
\left\{\begin{array}{l}
\dot{x}=y \\
\dot{y}=-x+u
\end{array},|u| \leq \varepsilon\right.
$$

It admits the classical first integral $V=\frac{1}{2}\left(x^{2}+y^{2}\right)$. The control which maximizes the derivative of $V$ under the constraint $|u| \leq \varepsilon$ is given by $u^{*}=-\varepsilon \operatorname{sgn} y$. It is discontinuous on the axis $\{y=0\}$ and gives rise to the following closed-loop system:.

$$
\left\{\begin{array}{l}
\dot{x}=y \\
\dot{y}=-x-\varepsilon \operatorname{sgn} y
\end{array},\right.
$$

In the sense of Filippov, solutions of the system (13) are solutions of the differential inclusion

$$
\left\{\begin{aligned}
\dot{x} & =y, \\
\dot{y} & \in \begin{cases}\{-x-\varepsilon \operatorname{sgn} y\} & \text { if } y \neq 0 \\
{[-x-\varepsilon,-x+\varepsilon]} & \text { if } y=0\end{cases}
\end{aligned}\right.
$$

Hence the equilibrium points of the discontinuous dynamical system (13) are $y=0$ and $x \in[-\varepsilon, \varepsilon]$ and all trajectories converge to the set of equilibrium points.

These "spurious" equilibrium points cannot be avoided with a discontinuous control. It is a drawback, but still allows practical stabilization on this example. However, the set $W$ of discontinuity points of the right hand side is not confined to a neighborhood of the target and it not clear, for more general systems, that the chattering solutions do not also appear far from it. We prove here that the classical Jurdjevic Quinn conditions prevent this from happening.

The natural notion (it corresponds to the solutions one observes in simulations or in reality) of solutions for differential equations with discontinuous right-hand side is the one of Filippov solutions; it consist in substituting to $\dot{q}=h^{\varepsilon}(q)$ a differential inclusion (its precise definition is recalled below, in section 4.2) that is single valued at points where $h^{\varepsilon}$ is continuous but multi-valued on $W$, i.e. at points where $h^{\varepsilon}$ is 
discontinuous. This extension of the right-hand side is theoretical process that gives rise to solutions called "chattering" ones; it introduces solutions that are not only the "naive" ones, and may defeat stability, or asymptotic stability (convergence).

It is clear that, in the sense of Filippov, the control $u^{\varepsilon}$ stabilizes the target orbit in the sense of Lyapunov because, for all $\alpha>0$, the set $\{V \leq \alpha\}$ is invariant. Moreover although we do not have asymptotic stability, the following result holds (we denote by $\mathcal{O}_{\delta} \Gamma$ the set $\left.\{q \in M, d(q, \Gamma)<\delta\}\right)$ :

Theorem 2 Let $h^{\varepsilon}$ be defined by (10).

- For any $\varepsilon \geq 0$, the orbit $\Gamma$ is stable for the closed-loop system $\dot{q}=h^{\varepsilon}(q)$.

- For any compact set $K$ and any neighborhood $\mathcal{O}_{\delta} \Gamma$ of the closed curve $\Gamma$, there is an $\varepsilon_{0}>0$ such that the closed loop system $\dot{q}=h^{\varepsilon}(q)$ has the following property if $\varepsilon \leq \varepsilon_{0}$ :

Any solution q, in the sense of Filippov, such that $q(0) \in K$, enters $\mathcal{O}_{\delta} \Gamma$ in finite time, and remains inside it for large times.

The proof is given in section 4.2 below.

\section{Proofs}

\subsection{Differential inclusions and invariance theorem}

We recall in this section a LaSalle invariance principle for differential inclusions due to A. Bacciotti and F. Ceragioli [BAC 99]. We give a simpler version of the Theorem 3 for continuously differentiable Lyapunov functions, which is sufficient for our purpose. To state this theorem we recall some basic definitions, for a general presentation of the theory of differential inclusion the reader is referred to [FIL 88].

Let $F$ be an upper semi-continuous multivalued map with compact, convex values and consider the differential inclusion,

$$
\dot{x} \in F(x) .
$$

A solution of (15) on a non-degenerate interval $I \subset \mathbb{R}$ is an absolutely continuous functions in $I$ such that $\dot{\phi}(t) \in F(\phi(t))$ for almost all $t \in I$. We denote by $S_{x_{0}}$ the set of solutions of (15) such that $\phi(0)=x_{0}$. We say that a solution is maximal if $I=[0,+\infty[$.

The set-valued derivative of a smooth function $V: \mathbb{R}^{n} \rightarrow \mathbb{R}$ with respect to (15) is defined as

$$
\dot{V}(x)=\{d V(x) \cdot v, v \in F(x)\} .
$$

Definition 3 A smooth Lyapunov function $V$ for (15) is a positive definite, continuously differentiable function such that

$$
\left.\left.\forall x \in \mathbb{R}^{n}, \dot{V}(x) \subset\right]-\infty, 0\right] .
$$


Definition 4 A set $\mathcal{W}$ is said to be a weakly invariant set for (15) if through each point $x_{0} \in \mathcal{W}$ there exists a maximal solution of (15) lying in $\mathcal{W}$.

Theorem 5 [BAC 99, Theorem 3] Let $V: M \rightarrow \mathbb{R}$ be a continuously differentiable Lyapunov function for (15). Let $\phi$ be a solution of (15) starting at $x_{0} \in V_{\alpha}$. Let

$$
\mathbb{W}=\left\{x \in \mathbb{R}^{n}, 0 \in \dot{V}(x)\right\},
$$

and let $\mathcal{W}$ be the largest weakly invariant set in $\overline{\mathbb{W}} \cap V_{\alpha}$. Then $d(\phi(t), \mathcal{W}) \rightarrow 0$ as $t \rightarrow+\infty$.

Note that the actual Theorem 3 in [BAC 99] is more powerful because is does not assume differentiability of $V$.

\subsection{Proof of Theorem 2}

Since $V$ is proper (5), take $\alpha>0$ such that $K \subset V_{\alpha}$. We consider the differential inclusion

$$
\dot{q} \in K h^{\varepsilon}(q),
$$

with $K h^{\varepsilon}(q)$ defined by (12).

Define $\psi^{\varepsilon}$ by $\psi^{\varepsilon}(q)=\sum_{i=1}^{m} u_{i}^{\varepsilon}(q) f_{i}(q)$, so that $h^{\varepsilon}=f_{0}+\psi^{\varepsilon}$. Since $f_{0}$ is continuous and $\psi^{\varepsilon}$ is locally bounded,

$$
K h^{\varepsilon}(q)=f_{0}(q)+K(\psi)(q) .
$$

We recall $W=\left\{q \in V_{\alpha} \backslash \mathcal{O}_{\delta} \Gamma, L_{f_{i}} V=d V . f_{i}=0\right\}$. It is a smooth $m$-dimensional submanifold of $\mathbb{R}^{n}$. Let us compute $K \psi^{\varepsilon}(q)$. For $q \in W$,

$$
\begin{aligned}
K \psi^{\varepsilon}(q) & =\bigcap_{\rho \in \mathbb{N}} \overline{\mathrm{co}}\left\{\left(\sum_{i=1}^{m} u_{i}^{\varepsilon} f_{i}\right)(B(q, 1 / \rho) \backslash W)\right\}, \\
& =\left\{\sum_{i=1}^{m} u_{i} f_{i}(q), \sqrt{\sum_{i=1}^{m} u_{i}^{2}} \leq \varepsilon\right\},
\end{aligned}
$$

while, for each point $q \notin W, \psi^{\varepsilon}($.$) is continuous at q, K \psi^{\varepsilon}(q)=\left\{\psi^{\varepsilon}(q)\right\}$.

The set value derivative of $V$ with respect to (18) is reduced to a point,

$$
\dot{V}^{\varepsilon}(q)=\left\{-\sqrt{\sum_{i=1}^{m} L_{f_{i}} V(q)^{2}}\right\} .
$$

Indeed it is obtained by basic calculus if $q \notin W$ and if $q \in W$, then by (4) and (20) for all $v \in K h^{\varepsilon}(q), d V(q) \cdot v=0$. Hence, the set of discontinuity $W$ (7) of the the discontinuous vector field (10) and the zero set of the set value derivative $\mathbb{W}$ (17) of the differential inclusion (18) are equal.

By the Theorem 5, if $\phi(0) \in V_{\alpha}$, then for all $t \geq 0, \phi(t) \in V_{\alpha}$, more over the distance $\mathrm{d}(\phi(t), \mathcal{W})$ tends to zero as $t$ tends to infinity, where $\mathcal{W}$ is the largest weakly invariant set in $W \cap V_{\alpha}$. Then the Theorem 2 is a direct consequence of the Lemma 6 . 
Lemma 6 Assume that the conditions (2), (4), (5) and (6) are satisfies, then for any compact set $K$, there exists $\varepsilon_{0}$ such that for all $\varepsilon \leq \varepsilon_{0}$, the largest weakly invariant set of $(18)$ is in $\mathcal{O}_{\delta} \Gamma$.

Proof of Lemma 6 If the set $W$ is empty then the lemma is trivial. Assume that $W$ is not empty. The idea is to partitioned the set $V_{\alpha} \cap W \backslash \mathcal{O}_{\delta} \Gamma$ into subsets on which the drift $f_{0}$ is transversal. Note that on the orbit $\Gamma$ (3) the differential of $V$ cancels (6).

Let $W_{k}(21)$ be the subset of $V_{\alpha} \backslash \mathcal{O}_{\delta} \Gamma$, where all the Lie bracket of length lower or equal to $k$ are in the kernel of the differential of $V$.

$$
W_{k}=\left\{q \in V_{\alpha} \backslash \mathcal{O}_{\delta} \Gamma, L_{\left(\operatorname{ad} f_{0}\right)^{s} f_{i}} V(q)=0,0 \leq s \leq k\right\},
$$

Note that $W_{0}=W \cap V_{\alpha} \backslash \mathcal{O}_{\delta} \Gamma$ and, because of the hypothesis (2), $W_{l}=\varnothing$. Then we define the set $W_{k}^{\tau}(22)$, subsets of $W_{k}$, where the vector field $f_{0}$ is transversal.

$$
W_{k}^{\tau}=\left\{q \in W_{k},\left|L_{\left(\text {ad } f_{0}\right)^{k+1} f_{i}} V(q)\right| \geq \tau\right\} .
$$

Since $W_{l}=\varnothing, \exists s<l, \exists \tau_{s}>0, W_{s}^{\tau_{s}}=W_{s}$ and $W_{s} \neq \varnothing$.

By induction, for small $\varepsilon$, there exists $\gamma_{i} \geq 0, i \in[0 . . s]$ such that, in a finite time shorter than $\left(\gamma_{0}+\ldots+\gamma_{s}\right)$, all trajectories of the differential inclusions (18) starting in $W_{0}$, goes out of $W_{0}$. In other word, there is no weakly invariant set in $W_{0}$.

Remark that by smoothness of $V$ and $f_{i}$, there exists $M>0$, such that for all $q \in V_{\alpha} \cap \mathcal{O}_{\delta} \Gamma, k \in[0 . . l]$ and $i \in[1 . . m],\left|L_{\left(\mathrm{ad} f_{0}\right)^{k} f_{i}} V(q)\right| \leq M$. Note that $M$ becomes bigger and bigger while $\delta$ tends to zero.

Lemma 7 Let $k \geq 0$ and $\delta_{k-1}=\left(\tau_{k}-m \varepsilon M\right) / 2 M$. Let $\phi($.$) be a solution of (18)$ starting in $W_{k}^{\tau_{k}}$. If $0<\varepsilon<\min \left(\tau_{k} /(M m), 1 / m\right)$ then

$-\forall t \in] 0,2 \delta_{k-1}\left[, \phi(t) \notin W_{k}\right.$.

- if $k \geq 1$ then $\left.\forall \tau_{k-1} \in\right] 0, M \delta_{k-1}^{2}\left[, \exists \gamma_{k-1} \in\right] 0, \delta_{k-1}[$, such that

$$
\forall t \in\left[\gamma_{k-1}, \delta_{k-1}-\gamma_{k-1}\right] \text {, either } \phi(t) \notin W_{k-1} \text { or } \phi(t) \in W_{k-1}^{\tau_{k-1}} .
$$

Proof of Lemma 7 We can compute, almost everywhere, the time derivative of the $k^{\text {th }}$ Lie derivative $t \mapsto L_{\left(\text {ad } f_{0}\right)^{k} f_{i}} V(\phi(t))$,

$$
\frac{d}{d t} L_{\left(\operatorname{ad} f_{0}\right)^{k} f_{i}} V(\phi(t)) \in\left\{L_{\left(\operatorname{ad} f_{0}\right)^{k+1} f_{i}} V(\phi(t))+d V(\phi(t)) . v, v \in K \psi(\phi(t))\right\},
$$

hence by the equality (19),

$$
\left|\frac{d}{d t} L_{\left(\operatorname{ad} f_{0}\right)^{k} f_{i}} V(\phi(t))\right| \leq(1+m \varepsilon) M \leq 2 M .
$$

Since $\phi(0) \in W_{k}^{\tau_{k}},\left|L_{\left(\operatorname{ad} f_{0}\right)^{k+1} f_{i}} V(\phi(0))\right| \geq \tau_{k}$ and $\left|L_{\left(\operatorname{ad~} f_{0}\right)^{k} f_{i}} V(\phi(0))\right|=0$. Then for all $t \geq 0$,

$$
\left|L_{\left(\operatorname{ad~} f_{0}\right)^{k+1} f_{i}} V(\phi(t))\right| \geq \tau_{k}-2 M t,
$$


in particular for

$$
\forall t \in\left[0, \tau_{k} / 2 M\left[,\left|L_{\left(\text {ad } f_{0}\right)^{k+1} f_{i}} V(\phi(t))\right|>0 .\right.\right.
$$

More over if $k \geq 1$, for all $t \geq 0,\left|\frac{d}{d t} L_{\left(\text {ad } f_{0}\right)^{k} f_{i}} V(\phi(t))\right| \geq \tau_{k}-2 M t-m \varepsilon M$, hence

$$
\left|L_{\left(\text {ad } f_{0}\right)^{k} f_{i}} V(\phi(t))\right| \geq\left(\left(\tau_{k}-m \varepsilon M\right)-M t\right) t
$$

in particular

$$
\forall t \in\left[\gamma_{k-1}, \delta_{k-1}-\gamma_{k-1}\right],\left|L_{\left(\text {ad } f_{0}\right)^{k} f_{i}} V(\phi(t))\right|>0 .
$$

\section{Conclusion}

To the best of our knowledge, this result (Theorem 2) was not stated in the literature.

We do not claim that, by itself, it is essential for applications: indeed, in practice, one prefers continuous feedback laws such as

$$
u_{i}^{\varepsilon}(q)=-\frac{\varepsilon}{\alpha} \min \left(\alpha, 1 /\left|L_{h} V\right|\right) L_{f_{i}} V,
$$

with $\alpha>0$ small and $\left|L_{h} V\right|$ defined as in (9). In a sense, the control law in (9) is an idealisation of these; it is also their limit as $\alpha \rightarrow 0$. Note however that this remark is not a way to prove Theorem 2; indeed, with these continuous control laws, no solution of the closed-loop differential equation stay in the set $W$ (see (7)) on any time interval, while when considering the "limit" control law (9), this phenomenon occurs; the proof of Theorem 2 consists in showing that, except in a neighborhood of the target orbit, where this is wrong, solutions eventually leave $W$ after "sliding" for some time. The same behavior for the continuous controls above results in different behavior depending on the situation for the "limit" discontinuous controls.

The motivation for those discontinuous control laws was rather that they allow some "homogeneity of degree zero" of the controls. This allows to give estimation on how the time needed to reach a neighborhood of the target $\Gamma$ varies as $\varepsilon$ goes to zero.

For instance, for low thrust transfer to a circular orbit, one can prove that the time taken using those discontinuous control laws is or the order of $1 / \varepsilon$, while it is not clear when using the continuous controls above. This is used in [BOM 06a]; that estimation can also be proved using the results in [BOM 06b], without referring to feedback laws.

Acknowledgements. This work was partially supported by Alcatel Alenia Space, Cannes, France, under a contractual research project. 


\section{References}

[BAC 99] Bacciotti A., Ceragioli F., "Stability and Stabilization of Discontinuous Systems and Nonsmooth Lyapunov Functions", ESIAM, vol. 4, 1999, p. 361-376.

[BOM 06a] BOMBRUN A., "Estimation of optimal time for low thrust transfers between elliptic orbits", AIMS' Sixth International Conference on Dyn. Systems, Diff. Equations and Applications, Poitiers, France, June 2006.

[BOM 06b] Bombrun A., Pomet J.-B., "On the Average Control System”, 17th International Symposium on Mathematical Theory of Networks and Systems (MTNS 2006), Kyoto, Japan, Jul. 2006.

[FIL 88] FILIPPOV V., Differential Equation with Discontinuous Right-hand side, Kluwer Academic Publishers, 1988.

[JUR 78] JURJEVIC V., QUINN J. P., "Controllability and Stability”, J. of Diff. Equations, vol. 28,1978 , p. 381-389.

[SON 98] Sontag E. D., Mathematical control Theory: Deterministic Finite Dimensional Systems, vol. 6 of Texts in applied mathematics, Springer, 1998. 